\title{
Phase transitions of a lattice-model for patchy particles with tetrahedral symmetry
}

\author{
N.G. Almarza, and E.G. Noya \\ Instituto de Química-Física Rocasolano, \\ CSIC, Serrano 119, E-28006 Madrid, Spain
}

(Dated: November 17, 2010)

\begin{abstract}
We present a simple lattice model which gives account of the low temperature phase equilibria of patchy particles with tetrahedral symmetry. Using such a model the phase diagram was computed as a function of the model parameters by means of Monte Carlo simulations. Depending on the details of the particle interactions a low-density ordered phase with diamond structure can appear as thermodynamically stable phase.

PACS numbers:
\end{abstract}




\section{INTRODUCTION}

Simple anisotropic models are representative of interactions in a large variety of systems, such a water[1-3], proteins[4-6] or anisotropic colloids, and both theoretical and simulation studies of those models have contributed to improve our understanding of the behaviour of such systems. For example, for proteins it has been recently shown that the metastable fluidfluid phase separation experimentally measured for several proteins can be quantitatively reproduced by simple anisotropic models[7].

During the last few years there has been a renewed interest in these simple models, which is partly motivated by recent advances in colloidal science that allowed the synthesis of colloids with anisotropic shapes or interactions $[8,9]$. Indeed it is now possible to design colloids with a quite accurate control on the location of the patches on their surface[10]. Theoretical and simulation studies on simple anisotropic models revealed that they can exhibit a complex phase behaviour[11-17].

The use of lattice models has proven to be very fruitful [18-28] in the analysis of the phase diagram of simple models with complex phase behaviour, with the advantage that simulations can be performed for larger system sizes with higher accuracy. For example, it has been shown that it is possible to design associating lattice models that reproduce some of the behaviour of water, including the density and diffusion anomalies[22, 27, 28]. Another interesting example is provided by a lattice model with two repulsive ranges, which predicts the existence of a body-centred-cubic (BCC) solid that exhibits reentrant melting, i.e. the BCC solid can melt either upon compression and upon expansion in a region of the phase diagram[24]. These two examples illustrate that simple lattice models are able to capture the complex behaviour of simple models.

In this work, we will compute the phase diagram of a lattice model that mimics the behaviour of patchy particles consisting on a spherical repulsive core with four interaction sites or patches in its surface with tetrahedral symmetry. The behaviour of tetrahedral particles has already been studied in the past using off-lattice models with the main objective of finding the appropriate conditions at which a solid with diamond structure could be obtained[29-33]. The interest in obtaining a colloidal crystal with diamond structure arises from its predicted optical band gap that makes it an ideal material for many applications in photonics[34-36]. 
The studies performed so far have shown that the phase diagram of tetrahedral particles exhibits multiple stable solid phases, that can include the diamond structure, a BCC solid, which is obtained by interpenetrating two diamond sublattices, and, at high pressures, a face-centred-cubic (FCC) structure. Both in the diamond and BCC solids the number of bonds is maximized, i.e. the four patches on a particle point directly at four nearest neighbours. As a consequence both solid structures have a similar potential energy. It has also been found that the stability of the diamond solid is very sensitive to the range of the potential[31-33], the stability of the diamond decreasing with respect to the BCC solid when the range of the potential increases and even disappearing from the phase diagram for long ranged models[33]. The stability of the diamond crystal for short ranged models has been attributed to the higher vibrational entropy of the diamond crystal as compared to that of the BCC solid[32, 33].

We will check how much of the complex behaviour of the tetrahedral models in the continuum can be captured by a lattice model. The use of a lattice model will allow us to explore in detail the phase diagram as a function of the parameters of the model. The results obtained for the lattice model will be compared to those obtained by Romano et al. [31, 32] and Noya et al.[33] using two different off-lattice models.

This paper is organized as follows: In the following section, we introduce our lattice model. In section III we describe the procedures to compute the phase diagram of the system, paying attention to the limits of both low and high temperature, and high density. In section IV the different phase diagrams are presented, and their topology is analyzed as a function of the parameters of the model. Finally in section $\mathrm{V}$ we discuss the analogies and differences between the results for lattice and off-lattice models and present the main conclusions of the work.

\section{THE MODEL}

The model proposed in this work will be denominated as the lattice patchy model (LPM) in reference to the off-lattice models that we try to mimic. It combines features of the socalled lattice hard sphere (LHS) models[20] with the orientationally dependent interactions of the three-dimensional associating lattice gas (ALG) model proposed by Girardi et al. [22, 28]

The model is defined on a simple cubic lattice with side length $L=8 n$, where $n$ is a 
positive integer. The sites on the lattice can be either occupied by one particle or empty. A hard-core interaction between particles excludes the simultaneous occupation of two sites $i$ and $j$ located at distances shorter than $r_{i j}<\sigma=2 \sqrt{3} d_{0}$, where $d_{0}$ is the distance between two nearest neighbours (NN) sites on the reference cubic lattice.

The total potential energy of the system can be calculated as:

$$
U=\sum_{i<j}\left[V_{L H S}\left(\mathbf{r}_{i j}\right)+V_{p}\left(\mathbf{r}_{i j}, S_{i}, S_{j}\right)\right]
$$

where $V_{L H S}\left(r_{i j}\right) / k_{B} T=\infty$ if $r_{i j}<\sigma$ and zero otherwise, with $k_{B}$ being the Boltzmann constant and $T$ the temperature. $V_{p}$ represents the patch interaction, which is similar to that proposed in the ALG model[22, 28]. In brief, each particle has four bonding arms (or four patches) distributed in a tetrahedral arrangement. The patch interaction is considered to occur only between particles separated by a distance $\mathbf{r}_{i j}=\sigma$. For $\sigma=2 \sqrt{3} d_{0}$, there are eight sites in the lattice at a distance $\sigma$ from a central occupied site that are located at positions $[ \pm 2, \pm 2, \pm 2] d_{0}$ with respect to the central site. Two possible configurations, $S_{i}= \pm 1$, are possible for the bonding arms, in which the four patches are pointing at directions $\{(111)(1 \overline{1} \overline{1})(\overline{1} 1 \overline{1})(\overline{1} \overline{1} 1)\}$ or $\{(\overline{1} \overline{1} \overline{1}),(11 \overline{1})(1 \overline{1} 1)(\overline{1} 11)\}$. This patch interaction can be expressed mathematically as:

$$
V_{p}\left(\mathbf{r}_{i j}, S_{i}, S_{j}\right)=\left\{\begin{array}{ll}
\epsilon_{0}-\left(\epsilon+\epsilon_{0}\right) \tau_{i}^{i j} \tau_{j}^{j i} & ; \text { for } r_{i j}=\sigma \\
0 & ; \text { for } r_{i j} \neq \sigma
\end{array} ;\right.
$$

where $\tau_{i}^{i j}$ is equal to one if there is a patch of particle $i$ pointing to particle $j$ and zero otherwise. In this work, we took $\epsilon>0$, i.e. the interaction between two particles, each one with a patch pointing to the other particle, is always attractive. The parameter $\epsilon_{0}\left(\left|\epsilon_{0}\right|<\epsilon\right)$ accounts for the interaction between two particles when one or both of them do not have a patch pointing to the other particle.

All throughout this paper reduced units are used. Energies will be given in units of $\epsilon$ and distances are given in units of $d_{0}$.

\section{A. Justification of the model}

The phase diagram of the ALG model on a BCC lattice has already been computed[28]. It has been found that it stabilizes a low density and a high density solid which are the 
lattice analogues of the diamond and BCC solids found for the off-lattice models that we try to mimic[31-33]. However, at high temperatures, transitions between the low density and high density ordered structures and between the fluid and high density solid become continuous, something that does not happen for the off-lattice tetrahedral models[28]. This can be avoided by including a hard-core in the interactions using the LHS model. The LHS model on a simple cubic lattice forms a BCC structure at close packing when the exclusion parameter $\sigma$ is a multiple of $\sqrt{3} d_{0}$. The LHS models with $\sigma^{*}=\sigma / d_{0}=\sqrt{3}$ and $\sigma^{*}=2 \sqrt{3}$ are known to exhibit a discontinuous solid-fluid transition (order-disorder)[20]. The BCC structure formed can contain a number of defects if their densities are less than the corresponding close packing densities, which are $\rho_{0} d_{0}^{3}=1 / 4$ for $\sigma^{*}=\sqrt{3}$, and $\rho_{0} d_{0}^{3}=1 / 32$ for $\sigma^{*}=2 \sqrt{3}$. The density of solid phase at the transition increases considerably when increasing the value of $\sigma$, being $\eta_{s} \simeq 0.52$ for $\sigma^{*}=\sqrt{3}$, and $\eta_{s} \simeq 0.94$ for $\sigma^{*}=2 \sqrt{3}$ (see Ref. 20). Here and throughout the paper the densities are expressed as packing fractions $\eta=\rho / \rho_{0}$, where $\rho=N / V$ is the density $\left(V=L^{3}\right.$ is the volume of the system, i.e., the number of sites in the underlying lattice) and $\rho_{0}$ is the density at close packing.

For the model potential described above, we expect that at high temperature the system will behave as the corresponding LHS model, whereas at low temperature the patch interactions will influence the phase behaviour. The known phase behaviour of the ALG and LHS models makes advisable, therefore, to consider in addition to the anisotropic interactions of the ALG model some excluded volume effects in order to attain a lattice model with phase behaviour resembling that of the off-lattice tetrahedral patchy models. Moreover the choice of $\sigma^{*}=2 \sqrt{3}$, was found to be required (instead of $\sigma^{*}=\sqrt{3}$ ) to assure that the density of the BCC phase stays well above that of the low density solid at any temperature. If either the ALG model[27] or $\sigma^{*}=\sqrt{3}$ version of our model are used, then one finds that the transition between the low density and the high density solids becomes continuous at some temperature range.

\section{COMPUTATION OF THE PHASE DIAGRAM}

In this section we present the procedures used in the computation of the phase diagram, and the corresponding results. The phase diagram was computed using several simulation techniques that include Grand Canonical Monte Carlo, Grand Canonical Wang-Landau, 
and Gibbs-Duhem-like integration (GDI). The essential features of the methodology can be found elsewhere $[24,25,37,38]$ and thus only a brief description is given here.

Given the relative complexity of the phase diagram, we first pay attention to the transitions occurring in the limits of close packing, and of high and low temperature, and the order-disorder transitions occurring at high density. Later we will focus on the global computation of equilibria between the fluid, low density solid, and high density solid phases at intermediate temperatures.

\section{A. The system at close packing}

At close packing conditions, the model considered in this work becomes equivalent to the ALG model at full occupancy. In the limit of $T \rightarrow \infty$ the interactions are dominated by the repulsive core and the orientation of the patches is random (between the two possible discrete orientations), whereas at $T=0$ the system is orientationally ordered, with each particle forming bonds (patch-patch interaction) with four of its eight neighbour particles located at a distance $\sigma$. The average potential energy per particle in these two limit cases is given by:

$$
U / N=\left\{\begin{array}{lc}
-2 \epsilon+2 \epsilon_{0} ; \quad \eta=1, T=0 \\
-\epsilon+3 \epsilon_{0} ; \quad \eta=1, T=\infty .
\end{array}\right.
$$

Between these two limits the system exhibits an order-disorder transition at a finite temperature $T_{c}^{*}=k_{B} T_{c} / \epsilon$ that depends on the value of $\epsilon_{0}^{*}=\epsilon_{0} / \epsilon$.

The order-disorder transition at close packing can be computed by realizing that it is possible to map the system at full occupancy to the antiferromagnetic Ising model (AFIM) on the BCC lattice. The procedure is similar to that reported for a two dimensional model of self-assembled rigid rods[39]. The energy of the system at full occupancy can be calculated as a sum of the energies of $N$ four-particle plaquettes, with $N$ being in this context equivalent to the maximum number of particles $\left(N=N_{\max }\right)$.

In Fig. 1, we show how the four-particle plaquettes can be defined. The plaquette depicted in the figure is associated to the site labelled as 0 . All the plaquettes required to give account of the potential energy of the system can be built by translation of the origin of the plaquette (label 0) to each site of the BCC lattice. According to the definition of the orientation variables $S_{i}$, a particle on the plaquette has a patch oriented to one of its NNs. 


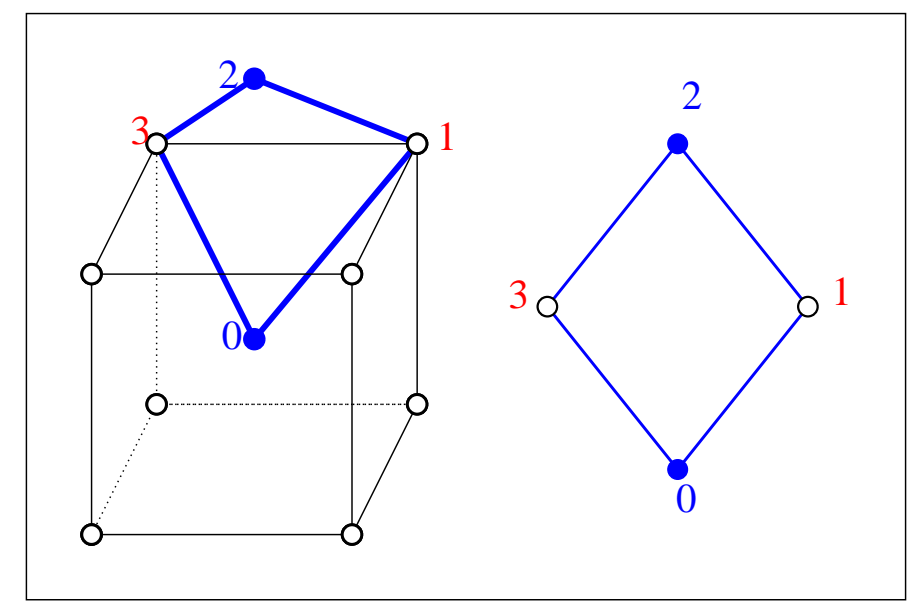

FIG. 1: Construction of four site plaquettes of the system. The coordinates of the labelled points in terms of the underlying lattice are $r_{0}=(0,0,0), r_{1}=(2,2,2), r_{2}=(0,0,4)$ and $r_{3}=(-2,2,2)$. On the right hand side, a projection of the plaquette (as seen from the front) is shown. Thick lines on the left panel (lines on the right panel) connect pairs of interacting sites.

If $S_{i}=1$ the patch on site $i$ points to the site $i+1$ (anticlockwise), whereas if $S_{i}=-1$ the patch points to the site $i-1$ (clockwise).

It is then straightforward to compute the energy of the plaquette as a function of the values of $S_{i}$ for the current model at $\eta=1$, and the corresponding values for the AFIM on the BCC lattice with $\mathrm{NN}$ interactions, and coupling constant $J[40]$. The results for representative configurations are gathered in table I.

TABLE I: Potential energy of four-site plaquettes in the LPM, and antiferromagnetic Ising model (AFIM) for representative plaquette configurations. The plus and minus signs correspond to orientations $S_{i}=+1$ and $S_{i}=-1$, respectively, for each of the four sites on a plaquette.

\begin{tabular}{|l|cccc|}
\hline \hline Plaquette & ++++ & +++- & ++-- & +-+- \\
\hline LPM & $4 \epsilon_{0}$ & $3 \epsilon_{0}-\epsilon$ & $3 \epsilon_{0}-\epsilon$ & $2 \epsilon_{0}-2 \epsilon$ \\
AFIM & $4 J$ & 0 & 0 & $-4 J$ \\
\hline \hline
\end{tabular}

The values of the potential energy in both models for a given plaquette configuration are related by:

$$
u_{p l a q}^{\mathrm{AFIM}}=\frac{4 J}{\epsilon+\epsilon_{0}} u_{p l a q}^{\mathrm{LPM}}+4 J \frac{\epsilon-3 \epsilon_{0}}{\epsilon+\epsilon_{0}} .
$$


Therefore the Helmholtz energies of the two systems are related by:

$$
A_{A F I M}\left(N, T_{\mathrm{I}}\right)=4 N J \frac{\epsilon-3 \epsilon_{0}}{\epsilon+\epsilon_{0}}+A_{L P M}(N, T)
$$

with:

$$
T=\frac{\epsilon+\epsilon_{0}}{4 J} T_{\mathrm{I}}
$$

Using the standard reduced temperatures for the AFIM and LPM as $T_{c}^{*(\mathrm{AFIM})}=$ $k_{B} T_{c}^{(\mathrm{AFIM})} / J$, and $T_{c}^{*}=k_{B} T_{c} / \epsilon$, respectively, the critical temperatures of both models are related by:

$$
T_{c}^{*}=\left(\frac{1+\epsilon_{0}^{*}}{4}\right) T_{c}^{*(\mathrm{~A} F I M)}
$$

Precise values of $T_{c}^{*(\mathrm{~A} F I M)}$ on the BCC lattice can be found in the literature[40].

\section{B. The limit of high temperature}

At high temperature, $k_{B} T>>\epsilon$, the phase behaviour of the system is basically controlled by the hard-core interactions. The LHS model has already been studied in Ref. 20. However, in order to check our programs and to get a more accurate value of the equilibrium conditions we have carried out a series of Grand-Canonical Wang-Landau simulations[25, 37] of the LHS model with $\sigma^{*}=2 \sqrt{3}$.

We have considered the following system sizes: $L=12,16,20,24,32,36$, and 40. For each system size estimates of the order-disorder transition have been obtained by computing the value of $\beta \mu(L)$ that maximizes the density fluctuations, $\mu$ being the chemical potential, and $\beta \equiv\left(k_{B} T\right)^{-1}$. Let us designate as $\beta \mu_{t}(L)$ such value. At those conditions we computed the average packing fraction $\eta_{m}(L)$ and the average fluctuation of the same quantity $\delta \eta(L)=$ $\left[<\eta^{2}>-<\eta>^{2}\right]^{1 / 2}$. The results are presented in table II.

The finite-size results for $\beta \mu_{t}(L), \eta_{m}(L)$, and $\delta \eta(L)$ for systems of $L \geq 24$ are well represented by linear functions of the inverse of the volume;

$$
y_{t}(L)=y_{t}+a L^{-3}
$$

Using Eq. (8) we get the following estimates for the transition at the thermodynamic limit: $\beta \mu_{t}=2.8539(1), \eta_{m}=0.74326(1), \delta \eta=0.19873(1)$. The packing fractions of each phase in coexistence can also be obtained: $\eta_{f}=\eta_{m}-\delta \eta=0.5445(1), \eta_{s}=\eta_{m}+\delta \eta=0.9420(1)$. 
TABLE II: Estimates of the fluid-solid transition for the lattice hard sphere model with $\sigma^{*}=2 \sqrt{3}$ for different system sizes

\begin{tabular}{|ll|lll|}
\hline \hline$L$ & $N_{\max }$ & $\beta \mu_{t}(L)$ & $\eta_{m}(L)$ & $\delta \eta(L)$ \\
\hline 40 & 2000 & $2.849560(15)$ & $0.743096(3)$ & $0.198738(4)$ \\
36 & 1458 & $2.847931(11)$ & $0.743025(3)$ & $0.198740(2)$ \\
32 & 1024 & $2.845391(11)$ & $0.742931(2)$ & $0.198746(3)$ \\
28 & 784 & $2.841230(18)$ & $0.742765(3)$ & $0.198758(3)$ \\
24 & 432 & $2.833723(20)$ & $0.742455(4)$ & $0.198788(3)$ \\
20 & 250 & $2.819105(27)$ & $0.741881(5)$ & $0.198812(4)$ \\
16 & 128 & $2.786989(42)$ & $0.740899(7)$ & $0.198612(5)$ \\
12 & 54 & $2.704225(55)$ & $0.739716(6)$ & $0.196946(7)$ \\
\hline \hline
\end{tabular}

These results agree with those reported by Panagiotopoulos [20], which expressed in our units are $\beta \mu_{t}=2.86(2), \eta_{f}=0.545(2) ; \eta_{s}=0.942(2)$. Note that the chemical potential definition used in this work differs from that given in Ref. [20]. In our case in the limit of very low density: $\beta \mu \simeq \ln \left(N / L^{3}\right)=\ln \left(\rho d_{0}^{3}\right)$.

In the limit of high temperature: $\beta=0$, the canonical partition function of the LPM can be related to the canonical partition function of the LHS model as:

$$
Q_{L P M}(\beta=0, N, V)=2^{N} Q_{L H S}(N, V) .
$$

where $N \leq V / 32$. The factor $2^{N}$ appears due to the two possible orientations that a particle can exhibit in the patchy model. Therefore the chemical potential of the two models at the fluid-solid transition are related by:

$$
\beta \mu_{t}^{L P M}=\beta \mu_{t}^{L H S}-\ln 2 \simeq 2.1608 \pm 0.0001 .
$$

\section{Order-disorder transition of the high density solid}

Given the features of the model, and the limits at close-packing and high temperature, one expects that the BCC phase will undergo a transition between an orientationally ordered BCC solid (o-BCC phase) and a disordered BCC solid (d-BCC phase) . This transition is expected to be continuous, and like that at $\eta=1$, to belong to the three-dimensional Ising universality class. 
In order to compute the critical line, $\eta_{c}(T)$ or $\mu_{c}(T)$, using the standard simulation procedures $[41,42]$ it is very useful to find the appropriate order parameters. Firstly we can consider an order parameter to check whether the particles in the system exhibit a true BCC structure. This can be achieved by considering that the close packing BCC structure has a degeneracy $g=32$. Accordingly, it is possible to define 32 sub-lattices so that each one contains $L^{3} / 32$ sites and represents one of the 32 possible degenerate configurations of the system.

The lattice points are defined by three integers $\left(k_{x}, k_{y}, k_{z}\right)$ with $0 \leq k_{\alpha}<L$. Given a reference site $\mathbf{k}_{0}=\left(k_{0 x}, k_{0 y}, k_{0 z}\right)$, the points belonging to the same sublattice are given by:

$$
\left(k_{x}, k_{y}, k_{x}\right)=\left\{\begin{array}{cl}
\mathbf{k}_{0}+(4 l, 4 m, 4 n) & ; \text { Sites A }: t=+1 \\
\mathbf{k}_{0}+(4 l+2,4 m+2,4 n+2) & ; \text { Sites B }: t=-1
\end{array}\right.
$$

where $l, m$, and $n$ are integers. The reference points $\mathbf{k}_{0}$ are chosen to be those that fulfill $0 \leq k_{x} \leq 1,0 \leq k_{y} \leq 3,0 \leq k_{z} \leq 3$. The points on the sublattice are classified in two types according to the parameter $t$.

For a given configuration of the system, we define a BCC order parameter, $q$, as:

$$
q=\frac{N_{S L}^{\max }}{N}
$$

where $N_{S L}^{\max }$ is the number of particles in the most populated sublattice and $N$ is the total number of particles in the system. BCC phases are easily identified using $q$. For instance at the LHS limit the parameter $q$ of the BCC phase at the transition is $q \simeq 0.9987$. This high value of $q$ indicates that in the BCC solid most of the occupied positions are on the same sublattice, so that it is feasible to locate the order-disorder transition of the BCC lattice by considering a second order parameter, $m$, that takes into account exclusively the orientation of the particles staying at sites of the predominant sublattice. The parameter $m$ is defined as:

$$
m=\frac{\sum_{i} t_{i} S_{i}}{N_{S L}^{\max }}
$$

where the sum is carried out only over the sites of the predominant sublattice, and $S_{i}=0$ for empty sites on the sublattice. Values of $|m|$ close to one indicate that the particles in the BCC lattice are orientationally ordered (o-BCC), whereas values close to zero indicate that the BCC lattice is orientationally disordered (d-BCC).

In order to compute the critical line corresponding to the orientationally order-disorder transition of the BCC structure, we performed for different values of $\beta \mu$ a series of Grand 
Canonical Monte Carlo simulations around the expected critical temperature $T_{c}(\beta \mu)$, for several system sizes. The pseudo-critical temperatures for each system size $T_{c}(L, \beta \mu)$ can be estimated by following the behaviour of the fourth order Binder cumulant of the order parameter[42]: $g_{4}=<m^{4}>/<m^{2}>^{2}$, as $T_{c}(L, \beta \mu)$ is defined as the temperature at which $g_{4}$ reaches the universal value expected for the three dimensional Ising universality class for cubic boxes with periodic boundary conditions: $g_{4}^{c} \simeq 1.604$ [43]. The results for different system sizes at a given value of $\beta \mu$ can then be used to estimate the critical properties at the thermodynamic limit: $T_{c}(\beta \mu)$ and $\eta_{c}(\beta \mu)$; by fitting the simulation results using[41, 42]:

$$
\begin{gathered}
T_{c}(L, \beta \mu)=T_{c}(\beta \mu)+a L^{-(1+\omega) / \nu} . \\
\eta_{c}(L, \beta \mu)=\eta_{c}(\beta \mu)+a L^{-1 / \nu} .
\end{gathered}
$$

The values of the critical exponents, $\nu$ and $\omega$, were taken from Ref. [43]. In table III some points of the order disorder transition are given for different values of the non-patch interaction $\epsilon_{0}$.

\begin{tabular}{|c|c|c|c|c|c|c|}
\hline & $\epsilon_{0}^{*}=0$ & & & & & \\
\hline$\beta \mu$ & 0.5 & 1.0 & 1.5 & 2.0 & 2.5 & 3.0 \\
\hline$T_{c}^{*}$ & $1.4541(7)$ & $1.5048(6)$ & $1.5370(4)$ & $1.5567(5)$ & $1.5687(4)$ & $1.5766(4)$ \\
\hline \multirow[t]{2}{*}{$\eta_{c}$} & $0.9260(2)$ & $0.9548(1)$ & $0.9723(1)$ & $0.9830(1)$ & $0.9896(1)$ & $0.9937(1)$ \\
\hline & $\epsilon_{0}^{*}=0.2$ & & & & & \\
\hline$\beta \mu$ & 1.5 & 2.0 & 2.5 & 3.0 & 3.5 & \\
\hline$T_{c}^{*}$ & $1.7638(8)$ & $1.8192(7)$ & $1.8523(5)$ & $1.8736(8)$ & $1.8860(5)$ & \\
\hline \multirow[t]{2}{*}{$\eta_{c}$} & $0.9356(2)$ & $0.9610(1)$ & $0.9762(1)$ & $0.9854(1)$ & $0.9911(1)$ & \\
\hline & $\epsilon_{0}^{*}=0.8$ & & & & & \\
\hline$\beta \mu$ & 3.0 & 3.5 & 4.0 & 4.5 & 5.0 & \\
\hline$T_{c}^{*}$ & $2.6504(4)$ & $2.7367(11)$ & $2.7852(10)$ & $2.8141(9)$ & $2.8320(7)$ & \\
\hline$\eta_{c}$ & $0.9374(1)$ & $0.9637(1)$ & $0.9782(2)$ & $0.9867(1)$ & $0.9919(1)$ & \\
\hline
\end{tabular}

TABLE III: Estimates of the order-disorder transition for the BCC lattice

The critical lines given in Table III can be fitted to equations of the form:

$$
T_{c}(\eta)=T_{0}+a_{1}(1-\eta)+a_{2}(1-\eta)^{2} .
$$


TABLE IV: Fits of the critical lines for the order-disorder transition of the BCC solid. In the last column we include estimates computed from Eq. (7) in conjunction with data for the Ising model in a BCC lattice taken from Ref. [40]. The second column, labelled as $T_{c}^{*}(\eta=1)$, refers to the value of $T_{c}^{*}$ in the limit of $\eta \rightarrow 1$. This coincides with the $T_{0}$ parameter, but its value is given separately to indicate the statistical error in this limit.

\begin{tabular}{||c|ccccc||}
\hline \hline$\epsilon_{0}^{*}$ & $T_{c}^{*}(\eta=1)$ & $T_{0}$ & $a_{1}$ & $a_{2}$ & $T_{c}^{*}(\eta=1)[\operatorname{Ref}[40]]$ \\
\hline 0.00 & $1.5885(16)$ & 1.5885 & -1.8903 & 0.9975 & 1.5885 \\
0.20 & $1.906(3)$ & 1.9062 & -2.2805 & 1.0902 & 1.9063 \\
0.80 & $2.860(6)$ & 2.8599 & -3.4653 & 1.8942 & 2.8594 \\
\hline \hline
\end{tabular}

The parameters of the fits to Eq. 16 obtained for different values of $\epsilon_{0}^{*}$ are given in Table IV. The value of the critical temperature at close packing (extrapolated using Eq. (16) for $\eta=1$ ) can be used to check the consistency of the data by comparing with the result obtained from the mapping to the AFIM in a BCC lattice, for which $T_{c}^{*(A F I M)} \simeq 6.3542[40]$, using Eq. (7). Note that on a BCC lattice the order-disorder transition is coincident for the ferromagnetic Ising model and the AFIM. As shown in Table IV the agreement between the results from both routes is excellent.

\section{The limit of low temperature}

The most stable phase at some given thermodynamic conditions corresponds to that that minimizes the Grand Potential $\Phi$ :

$$
\Phi=U-\mu N-T S
$$

with $S$ being the entropy. At $T=0$ the Grand Potential reduces to[28]:

$$
\Phi=U-\mu N
$$

which can be divided by $N_{\max }$ at both sides to obtain:

$$
\phi=U / N_{\max }-\mu \eta
$$

The plausible phases at zero temperature are a diluted gas, an ordered diamond phase, and the ordered BCC phase. It is straightforward to compute the Grand Potential for each 


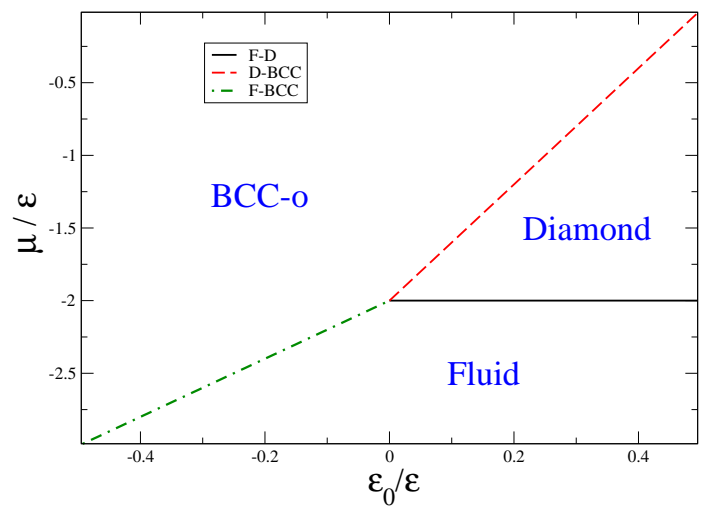

FIG. 2: Stable phases as function of $\mu$ and $\epsilon_{0}$, at zero temperature. o-BCC means ordered BCC solid.

one of these phases at zero temperature: $\phi_{g}(\mu)=0, \phi_{D}(\mu)=-\epsilon-\mu / 2$, and $\phi_{B C C}=$ $-2 \epsilon+2 \epsilon_{0}-\mu$. Phase transitions can then be simply calculated by equating the Grand Potential of these phases, from which the chemical potential at coexistence can be obtained. In Figure 2, the stability regions for the three considered phases at very low temperatures are shown as a function of the parameter $\epsilon_{0} / \epsilon$ that controls the non-patch interactions in the model. The diamond phase becomes the thermodynamically stable phase at intermediate values of the chemical potential (or pressure) for $\epsilon_{0}>0$.

\section{E. Phase transitions at intermediate temperatures}

The discontinuous transitions between the gas (fluid), the diamond and the BCC solid at finite temperatures have been calculated by a GDI. The variable of integration was chosen by numerical convenience in each region of the phase diagram. At low temperatures, we apply the differential equations given by:

$$
d \mu=\left(\frac{\mu}{T}-\frac{\Delta U}{T \Delta N}\right) d T
$$

and

$$
d T=\left(\frac{\mu}{T}-\frac{\Delta U}{T \Delta N}\right)^{-1} d \mu,
$$

whereas in the integrations from the high temperature limit we used:

$$
d(\beta \mu)=\frac{\Delta U}{\Delta N} d \beta
$$


where $\Delta$ indicates the difference of the value of a given property in the two phases at equilibrium.

The integration of the above differential equations was carried out using the fourth-order Runge-Kutta method. We have checked the consistency of the results by performing GDI from both the low and high temperature limits. The calculations shown in the figures were performed using systems of size $L=40\left(N_{\max }=2000\right)$. The system size dependence was checked by conducting calculations also for $L=24$, and $L=32$. No significant differences were found between the results for different system sizes.

In order to avoid the possible effects that the continuous order-disorder transition in the BCC solid might have on the gas-BCC coexistence curve, small values of the integration step $\delta(\beta)$ were considered in the GD integration from $\beta=0$. For all the cases considered, a good coalescence of different curves at triple points was obtained, which is a signature of the accuracy of the GDI results.

The GDI results were also checked by comparing the coexistence points with those obtained from Grand Canonical Wang Landau simulations at some selected temperatures[24, $25]$.

\section{PHASE DIAGRAMS}

Using the techniques described in previous sections we have computed the phase diagram of the model for several values of $\epsilon_{0}^{*}$. The topology of the phase diagrams can be classified in four types. We will describe them in order of growing complexity.

\section{A. Attractive non-patch interaction $-1<\epsilon_{0}^{*}<0$}

In Figure 3 the $T-\eta$ projection of the phase diagram for $\epsilon_{0}^{*}=0.00$ is shown. This diagram is representative of the model in the range $-1<\epsilon_{0}^{*}<0$, i.e., for attractive nonpatch interactions. At all temperatures there is a phase transition between a translationally disordered phase (labelled as fluid), and a high density phase with translational order. In the high density phase, particles are basically ordered in a BCC structure (most of the particles occupy sites of the predominant BCC sublattice). At densities below $\eta=1$, some defects are present in the system, mostly vacant sites on the BCC sublattice, but also some 
displaced particles. Paying attention to the orientational degrees of freedom, the BCC solid undergoes a phase transition at intermediate temperatures. At low temperature the particles are oriented in such a way that the patch-patch orientation is favored. The bond framework defined by the patch-patch interaction can be interpreted in terms of two diamond structures [28]. The orientational transition between the low and high temperature BCC phase is found to be continuous, defining a critical line starting at $\eta=1$ and ending when it meets the $\mathrm{BCC}$ branch of the fluid-BCC transition.

A low density phase with diamond structure is not stable in any region of the phase diagram for attractive non-patch interaction. The absence of an energy penalty associated to the interpenetration of the two diamond sublattices to obtain a BCC structure favours the stability of BCC over the diamond structure due to the higher density of the former. In the particular case of $\epsilon_{0}^{*}=0$ the diamond and BCC solids are degenerate at $\mu / \epsilon=-2$ and zero temperature (see Fig.2). However at any finite temperature the diamond is never stable, so that at finite temperature the phase diagram for $\epsilon_{0}^{*}=0$ is representative of the behaviour for $\epsilon_{0}^{*}<0$.

\section{B. Slightly repulsive non-bonding interaction $0 \leq \epsilon_{0}^{*}<0.71$}

As it can be seen in Figure 2 for $\epsilon_{0} \geq 0$ the diamond phase becomes stable in a certain range of chemical potentials at $T=0$. At finite temperatures this translates into a certain range of temperatures at which the diamond phase is thermodynamically stable. As one increases further the temperature, the low entropy of this phase makes it unstable with respect to the fluid-BCC equilibrium. In Figure 4 we show a typical phase diagram of this region. The stability of the diamond phase ends at a triple point in which fluid, diamond and ordered BCC phases coexist. As for the case $\epsilon_{0}<0$ the BCC phase exhibits an orientational order-disorder transition, which is shifted to higher temperatures, with a dependence for fixed packing fraction that can be approximately described by $T_{c}(\eta) \propto\left(1+\epsilon_{0}^{*}\right)$.

An interesting result is that a small non-patch repulsion (i.e., a small positive value of $\left.\epsilon_{0}^{*}\right)$ is enough to get a considerable temperature range of stability of the diamond phase. For instance, the triple point corresponding to the largest temperature with diamond stability appears at $T_{T P}^{*} \simeq 0.293$ for $\epsilon_{0}^{*}=0.01$, whereas for $\epsilon_{0}^{*}=0.001, T_{T P}^{*} \simeq 0.225$. 


\section{Medium repulsive non-bonding interaction $0.710 \leq \epsilon_{0}^{*}<0.90$}

As the value of $\epsilon_{0}$ increases from $\epsilon_{0}^{*}=0.71$, some qualitative changes happen in the phase diagram. In addition to the aforementioned increase of the stability region of the diamond phase, and the displacement of the order-disorder transition of the high density solid to higher temperatures, the density of the fluid branch of the high temperature fluid-BCC equilibrium increases with $\epsilon_{0}$. As a direct consequence this fluid density can reach values similar to or greater than the density range of the stability of the diamond phase $(\eta \simeq$ 0.5) when approaching the temperature region where diamond phase is thermodynamically stable. As a result, one can find for $\epsilon_{0}^{*} \geq 0.71$ a stable fluid denser than the diamond phase. This is illustrated in the phase diagram represented in Figure 5. It can be seen that there is a certain temperature range where the diamond phase can melt either by increasing or reducing the chemical potential (or the pressure). In other words, for the present model, the low density solid phase can melt under isothermal compression. This is usually known as reentrant melting and it occurs in real associating liquids (like water)[44]. For the off-lattice tetrahedral models that we try to mimic, reentrant melting have also been observed when the range of the interactions is below a given value[32]. Reentrant melting have also been observed for other lattice models[24, 25].

\section{Largely repulsive non-bonding interaction $\epsilon_{0}^{*}>0.90$}

If the value of $\epsilon_{0}$ is further increased, then one finds that the features of the phase diagram described for medium repulsive non-bonding interaction remain qualitatively unchanged in both the low and high temperature regions, however the GDI results indicate that further phases can appear. When integrating the fluid-BCC coexistence lines it is found that at intermediate temperatures the gas phase eventually undergoes a phase transition to a new

phase of lower energy, with an intermediate density between those of the gas phase and the high density solid. We have found it difficult to characterize this phase, since no reproducible results are found when dealing with different system sizes. This inconsistency might be due to incomensurability effects between the system sizes used in the simulation and the required dimensions to get the new phase stabilized. From the physical point of view the presence of additional phases could be interpreted as follows: in the region of low temperature the phase 


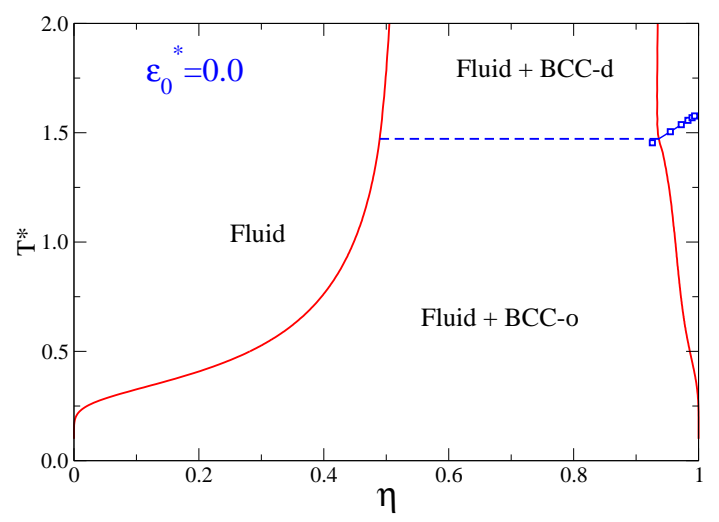

FIG. 3: Phase diagram for $\epsilon_{0}^{*}=0$. o-BCC means ordered BCC solid and d-BCC disordered BCC solid.

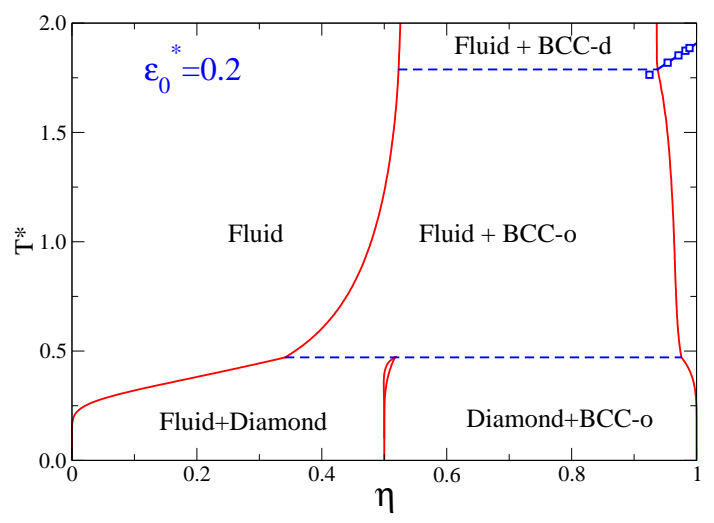

FIG. 4: Phase diagram for $\epsilon_{0}^{*}=0.20$. Labelling of regions as in previous figures.

behaviour is basically controlled by the attractive patch-patch interactions, whereas at the high temperature limit the hard core interactions are responsible for the phase transitions. At intermediate temperatures, and for high values of $\epsilon_{0}$, the interaction energy between pair of particles separated by a distance $2 \sqrt{3} d_{0}$ are still relevant, but the role of entropy makes that this interaction becomes on average repulsive. Under this view one can guess that the system could find thermodynamic stability by looking for configurations with distances between neighbour particles slightly above $2 \sqrt{3} d_{0}$. The search of the structure of such possible phases, besides being a difficult task, is out of the scope of the present work. 


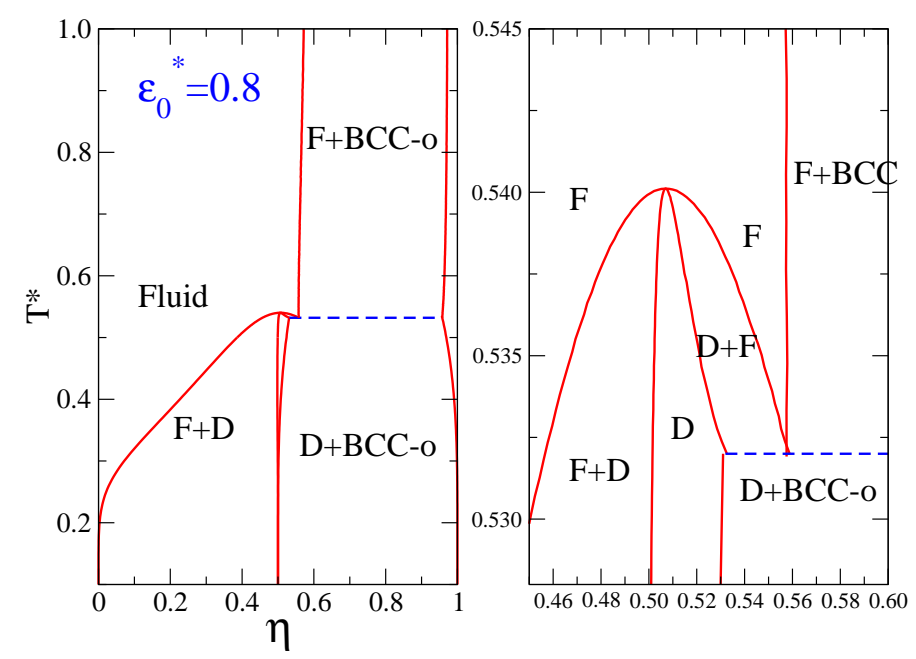

FIG. 5: Phase diagram for $\epsilon_{0}^{*}=0.80$. Labelling of regions as in previous figures, D means diamond phase, and F fluid phase.

\section{CONCLUSIONS}

The phase diagram of a lattice patchy model that combines a repulsive core plus four attractive sites in a tetrahedral arrangement has been calculated by computer simulation. This model was chosen to mimic the behaviour of off-lattice models of tetrahedral particles. The use of a lattice model has allowed us to explore a wide range of parameters of the model. Keeping the patch-patch interactions attractive $(\epsilon=1)$, non-patch interactions varied from attractive $\left(\epsilon_{0}=-1.0\right)$ to highly repulsive $\left(\epsilon_{0}=1.0\right)$. It was found that for attractive non-patch interactions only a high density solid is obtained with BCC structure that is orientationally ordered at low temperatures and orientationally disordered at high temperatures. When the repulsion is increased, reentrant behaviour is found in the fluid-low density solid coexistence line. A low density solid with diamond structure only becomes stable for repulsive non-patch interactions. Interestingly a quite small repulsion is enough to stabilize the diamond solid on a quite large region of the phase diagram. This suggests that adding an isotropic repulsion beyond the hard core of the colloidal particles could be used in experiments to stabilize the diamond structure.

When comparing the lattice patchy model with the models of tetrahedral particles in the continuum that have been studied by Romano et al.[31, 32] and by Noya et al.[33], both would correspond to non-interacting or slightly attractive non-patch interactions if one looks at the model potential. Both consist of a repulsive core plus four attractive 
sites and neither of them include non-patch interactions that disfavour the formation of a BCC structure. However, at finite temperatures, it has been found that the diamond structure can be stabilized over the BCC solid for short ranged models due to the higher vibrational entropy of the diamond structure. The interactions between the two diamond sublattices in the BCC reduce its vibrational entropy and this effect is more pronounced for short ranged models, for which the distance between the two sublattices decreases. The simple lattice model described here neglects the vibrational entropy. However, the lower vibrational entropy of the BCC solid can be mimicked by introducing a repulsion energy between the two sublattices, which in our model implies a positive value of $\epsilon_{0}$. Obviously this is an oversimplification because entropic effects become larger with temperature, something that can not be picked up with an energy penalty term. Following this recipe, long range off-lattice models are mapped by the lattice model with attractive non-patch interactions whereas short range models in the continuous can be related to positive values of $\epsilon_{0}$, the value of $\epsilon_{0}$ increasing with decreasing the range of the potential. It can be seen that with this mapping the phase diagram of the lattice model bears some resemblance with the low temperature region of off-lattice models. For attractive non-patch interaction the diamond is absent in the phase diagram, similarly to long range patchy models in the continuum[33]. For low repulsive non-patch interactions the diamond is stabilized, mimicking the behaviour of patchy models when the range is decreased[31,33]. Finally, the reentrant melting of the diamond structure that it is observed for very short ranged models in the continuum also appears for lattice models with medium repulsive non-patch interactions[32].

Obviously the underlying lattice imposes constraints and there are other features of the phase diagram that cannot be reproduced, which in this case correspond mainly to the high temperature and high density limits and are of less interest. As an example, the lattice model considered in this work cannot form the FCC structure found at high pressures[32, 33] due to the constraints imposed by the lattice.

In summary, it has been shown that lattice models are able to reproduce much of the behaviour of continuous patchy particles. The advantage of lattice models is that due to its simplicity it is possible to explore the model parameter space more quickly and simulations can be performed for larger system sizes. 


\section{Acknowledgments}

The authors gratefully acknowledge the support from the Dirección General de Investigación Científica y Técnica under Grants No. MAT2007-65711-C04-04 and FIS2010-15502, from the Dirección General de Universidades e Investigación de la Comunidad de Madrid under Grant No. S2009/ESP-1691 and Program MODELICO-CM and from CSIC under Grant No. 200980I099.

[1] J. Kolafa and I. Nezbeda, Mol. Phys. 61, 161 (1987).

[2] C. Vega and P. A. Monson, J. Chem. Phys. 109, 9938 (1998).

[3] C. L. Dias, T. Ala-Nissila, M. Grant, and M. Karttunen, J. Chem. Phys. 131, 054505 (2009).

[4] R. P. Sear, J. Chem. Phys. 111, 4800 (1999).

[5] N. Kern and D. Frenkel, J. Chem. Phys. 118, 9882 (2003).

[6] J. Chang, A. M. Lenhoff, and S. I. Sandler, J. Chem. Phys. 120, 3003 (2004).

[7] H. Liu, S. K. Kumar, and F. Sciortino, J. Chem. Phys. 127, 084902 (2007).

[8] V. N. Manoharan, M. T. Elsesser, and D. J. Pine, Science 301, 483 (2003).

[9] Y.-S. Cho, G.-R. Yi, J.-M. Li, S.-H. Kim, V. M. Manoharan, D. J. Pine, and S. M. Yang, J. Am. Chem. Soc. 127, 15968 (2005).

[10] D. J. Kraft, J. Groenewold, and W. K. Kegel, Soft Matter 5, 3823 (2009).

[11] E. G. Noya, C. Vega, J. P. K. Doye, and A. A. Louis, J. Chem. Phys. 127, 054501 (2007).

[12] X. Li, J. D. Gunton, and A. Chakrabarti, J. Chem. Phys. 131, 115101 (2009).

[13] F. Sciortino, A. Giacometti, and G. Pastore, Phys. Rev. Lett. 103, 237801 (2009).

[14] A. Giacometti, F. Lado, J. Largo, G. Pastore, and F. Sciortino, J. Chem. Phys. 131, 174114 (2009).

[15] A. B. Pawar and I. Kretzschmar, Macromol. Rapid Commun. 31, 150 (2010).

[16] G. Doppelbauer, E. Bianchi, and G. Kahl, J. Phys.: Condens. Matter 22, 104105 (2010).

[17] J. M. Tavares, P. I. C. Teixeira, M. M. Telo da Gama, and F. Sciortino, J. Chem. Phys. 132, $234502(2010)$.

[18] A. Z. Panagiotopoulos and S. K. Kumar, Phys. Rev. Lett. 83, 2981 (1999).

[19] N. G. Almarza and E. Enciso, Phys. Rev. E 64, 042501 (2001). 
[20] A. Z. Panagiotopoulos, J. Chem. Phys. 123, 104504 (2005).

[21] A. Diehl and A. Z. Panagiotopoulos, Phys. Rev. E 71, 046118 (2005).

[22] M. Girardi, A. L. Balladares, V. B. Henriques, and M. C. Barbosa, J. Chem. Phys. 126, $064503(2007)$.

[23] H. C. M. Fernandes, J. J. Arenzon, and Y. Levin, J. Chem. Phys. 126, 114508 (2007).

[24] J. S. Høye, E. Lomba, and N. G. Almarza, Molec. Phys. 107, 321 (2009).

[25] N. G. Almarza, Capitán, J. A. Cuesta, and E. Lomba, J. Chem. Phys. 131, 124506 (2009).

[26] C. E. Fiore, M. M. Szortyka, M. C. Barbosa, and V. B. Henriques, J. Chem. Phys. 131, 164506 (2009).

[27] M. M. Szortyka, V. B. Henriques, M. Girardi, and M. C. Barbosa, J. Chem. Phys. 130, 184902 (2009).

[28] M. M. Szortyka, V. B. Henriques, M. Girardi, and M. C. Barbosa, J. Chem. Phys. 132, 134904 (2010).

[29] Z. Zhang, A. S. Keys, T. Chen, and S. C. Glotzer, Langmuir 21, 11547 (2005).

[30] J. P. K. Doye, A. A. Louis, I.-C. Lin, L. R. Allen, E. G. Noya, A. W. Wilber, H. C. Kok, and R. Lyus, Phys. Chem. Chem. Phys. 9, 2197 (2007).

[31] F. Romano, E. Sanz, and F. Sciortino, J. Phys. Chem. B 113, 15133 (2009).

[32] F. Romano, E. Sanz, and F. Sciortino, J. Chem. Phys. 132, 184501 (2010).

[33] E. G. Noya, C. Vega, J. P. K. Doye, and A. A. Louis, J. Chem. Phys. 132, 234511 (2010).

[34] M. Maldovan, C. K. Ullal, W. C. Carter, and E. L. Thomas, Nat. Mat. 2, 664 (2003).

[35] M. Maldovan and E. L. Thomas, Nat. Mat. 3, 593 (2004).

[36] A.-P. Hynninen, J. H. J. Thijssen, E. C. M. Vermolen, M. Dijkstra, and A. van Blaaderen, Nature Materials 6, 202 (2007).

[37] E. Lomba, C. Martín, N. G. Almarza, and F. Lado, Phys. Rev. E 71, 046132 (2005).

[38] E. Lomba, N. G. Almarza, C. Martín, N. G. Almarza, and C. McBride, J. Chem. Phys. 126, $244510(2007)$.

[39] N. G. Almarza, J. M. Tavares, and M. M. Telo da Gama, Phys. Rev. E (sumbitted) (2010).

[40] P. H. Lundow, K. Martström, and A. Rosengren, Phylosophycal Magazine 89, 2009 (2009).

[41] N. B. Wilding, Phys. Rev. E 52, 602 (1995).

[42] D. P. Landau and K. Binder, A Guide to Monte Carlo Simulations in Statistical Physics, 2nd edition (Cambridge University Press, 2005). 
[43] H. W. J. Blöte, E. Luijten, and J. R. Heringa, J. Phys. A: Math. Gen. 28, 6289 (1995).

[44] O. Mishima, J. Chem. Phys. 132, 234502 (1993). 